PROCEEDINGS OF THE NUTRITION SOCIETY

A Scientific Meeting was held at St Angela's College for Home Economics, Lough Gill, Sligo, Republic of Ireland on 6/7 September 1990

\title{
Symposium on 'Slimming'
}

\author{
Moderator's remarks \\ BY J. V. G. A. DURNIN \\ Institute of Physiology, University of Glasgow, Glasgow G12 $8 Q Q$
}

A symposium on obesity and slimming has probably more practical pertinence to the general population than almost any other nutritional topic I can quickly think of. However, except for people who are actually fat, or are in the process of slimming, or are making money out of people who are fat or slimming, obesity and slimming are really rather boring topics. There are so many unanswered simple questions, from the elementary one of 'do fat people eat excessively large quantities of food?', that it becomes quite frustrating to be confronted by all this ignorance. The ignorance is extensive in spite of the enormous amount of research carried out in the past two or three decades.

It is, thus, a measure of the success of the present symposium, in the choice of speakers, the material which they covered, and the high level of delivery and of interest generated, that I am sure that the great majority of the audience felt that their participation had been really worth while. The organizers are to be congratulated, not least for the generous hospitality.

Slimming is something about which most of the adult population in most of the industrialized world has been concerned, at least on occasions. There are few adults who reach late middle-age who have not, at a minimum, either actively considered the desirability of losing weight, or have, on occasion, restricted their food intake by refraining from eating some attractive morsel of food, or from having a modest refill of their wine glass, because they did not want to run the danger of an excess energy intake.

The problem of slimming is one not only of widespread and generally recognized importance but its investigation, in one way or another, has been going on for a very long time. It is about 40 years since the basic studies on appetite control were carried out by Mayer, Brobeck, Kennedy, and others, and since then there has been almost a logarithmic increase in the reporting of studies of various kinds on 'slimming'. However, in all these years, with all the distinguished brains which have been obsessed with the investigation of obesity, with all the millions and millions of pounds, dollars, 'punts', which have been spent, our discoveries have been very limited. Slimming exists as an antidote to obesity, which exists because energy intake is or was greater than energy expenditure, and we know little more than we did many years ago about why energy intake should be greater than expenditure. 
Part of the problem relates to our insecure baselines: we are not sure at what point slimming becomes desirable for reasons other than those simply related to a wish to lose weight mainly for cosmetic purposes. At what stages does body composition change so that the amount of fat becomes unphysiologically large and may be a danger to health?

Part of our uncertainty in this clinically important area reflects the inadequacy of the methodological and physiological information available. Methodological, because although there are many techniques available for determining fatness, they are often not utilized, and dependence is placed on various ratios of height and weight, which may not always indicate fatness. For reasons which to me seem basically peculiar to nutritionists, we are apparently compelled in any large-scale investigation to rely on only simple measurements; anything which requires more than the skill and ability of a mentally retarded infant is too difficult to be entertained as a field method. Therefore, we seldom measure fatness directly. Nor, even if we do, do we have agreed norms for fatness. In such a context, I do not think you can expect a critical scientist to be happy about accepting a body mass index (BMI) of, say, under 25 as depicting a desirable level of fatness (since BMI does not measure fatness); indeed, in individuals with presumably small muscle and skeletal masses, a BMI of 25 is quite compatible with considerable quantities of adipose tissue.

There is also an amazing dearth of information on the relationship of fatness to health (or ill-health), and perhaps a not-so-amazing lack of agreement on the implications for health of the limited information available. In spite of controlling the influence of all sorts of variables, smoking, age, sex, exercise habits, etc., we can still have basic disagreement about the relevance of moderate obesity to morbidity and mortality. Of course, I use the term 'moderate obesity' very loosely. What is moderate obesity? I think I know what I mean by that, but many people I suspect might not agree with me: the crucial fact is that it is not defined. We, therefore, have complicated decisions to take about how persuaded we are, on the basis of the large mass of data from Norway or from the American epidemiological data, that 'moderate obesity' (a BMI of 25-30?) either is almost completely unrelated to morbidity and mortality, or is a highly dangerous condition.

An even more controversial aspect of slimming lies in what are the effective methods, if any, of losing weight and keeping it lost. The fact that Rosemary Conley, on the basis of virtually no expert knowledge whatsoever (as she will freely admit) can write two or three extremely popular books in a couple of years, full of what most of us would consider of questionable validity but expressed in a way that obviously appeals to millions of women, epitomizes the lack of success and of respect for standard methods of treating obesity. Even taking into account the attractive title of her book, The Hip and Thigh Diet, with its instant appeal to the huge mass of ordinary plump women who are convinced that their shape is not really acceptable in present-day society, its success on such a scale could occur only in a situation where standard dietetic or medical advice was regarded as unlikely to fulfil the need of the recipient.

Even more controversial is the respective roles of dieting and exercise. Of course, it all depends on what is meant by exercise. Moderate walking for $0.5 \mathrm{~h}$ three times weekly may make the individual feel a little 'healthier' if previously he or she had been extremely inactive, but is unlikely to be very effective in slimming. On the other hand, if one looks at the army of dedicated joggers, the vast majority will almost invariably be slim, muscular people and there will rarely be an obese person among them. If you look 
carefully at a TV recording of the start of one of the popular marathons (the London, or Boston or whatever) everyone, without exception, is slim (at least those in the front rows). It is also quite easy to find out (although there is very little information on this that I know of) that these joggers have not always been slim and muscular, and many of them were previously heavier and fatter than they are now. The slimness is, therefore, the result of the exercise and has not always coincided with it.

There are several other areas of controversy in the subject of obesity and slimming. What is the importance of genetic factors? Our basal metabolism is certainly affected by our inherited characteristics. But are there other inherited influences which may make us more likely to become obese? Perhaps, our efficiency of digestion and absorption of foods? Does the type of foods that we eat affect our body composition, sugars, fats, alcohol, fibre? Does our particular eating pattern affect our dietary-induced thermogenesis?

Does the distribution of fat in the body, the proportion of total fat which is in the subcutaneous tissues or intra-abdominal, make our attempts at slimming likely to be more or less successful?

If we successfully slim by reducing markedly our total energy intake over a prolonged period, does this insidiously result in a semi-permanent diminution of our basal metabolic rate and, thus, induce a long-term requirement to maintain a lower energy intake than would previously have been the case?

Unfortunately, not many of these questions were directly dealt with in the symposium; quite understandably since the attempt at answers would be mostly speculative. However, I am delighted to say that in my ' 100 ' years of attending scientific symposia, rarely, if ever, have I listened to seven speakers all of whom gave clear, lucid, interesting, often humorous, and highly relevant presentations. However, I think it is obvious that we have still to collect much more precise information on the health risks of varying levels of fatness, and to obtain a clearer view of these risks, I think we require more comprehensive cross-sectional and longitudinal data on the two sexes, at different ages, where fatness is measured by more than just height and weight. We also need to study the interaction of that information with levels of habitual physical activity, and probably also with the traditional diet. I think, although I do not pretend to know, that the risks of an early death might not be the same for a moderately obese Greek or Southern Italian as they would be for a poor urban inhabitant of these islands.

We also have an important task to do educationally. If we believe, as I think many of us do, that moderate obesity has no significant health hazards for an otherwise healthy woman, we should try to get that message across very clearly, particularly to doctors who, I am afraid, are provided with very confusing advice about almost everything connected with obesity and slimming. Of course we know that health reasons may not be the primary ones in a woman's mind when she wants to slim. But if we follow in this country recent trends in the United States, it is clear that the 'health message' has become very firmly embedded in the minds of the middle-class population, often in the wrong way so that a BMI of 22 is propagated as indicating dangerous fatness in a woman. To undertake this educational role needs better contact with and understanding of the media.

I also think we should be able to discuss the complex role of exercise in slimming in a manner which does not have to rely on limited and largely anecdotal information. I have been making frequent visits to several places in the United States during the past 2 to 3 
years and I am struck by the fact that in middle-class residential districts, it would be only a slight exaggeration to say that there now seems to be not a range of female builds, from skinny to fat, but only two types. One type consists of very lean muscular women, who can be of almost any age, whom you see jogging or cycling: the other type is made up of very large women whom one sees waddling along the footpath. I have the impression that the thing which has resulted in the difference between these types is the combination of dieting and exercise, but with exercise playing a considerable part.

There is obviously material for many more symposia on slimming but this one, at least, was most successful and informative. 